\title{
Observations of solar flares with IRIS and SDO
}

\author{
D. $\mathrm{Li}^{1,2,3}$, D. E. Innes ${ }^{2}$, and Z. J. Ning ${ }^{1}$ \\ 1 Key Laboratory of Dark Matter and Space Science, Purple Mountain Observatory, CAS, Nanjing 210008, PR China \\ e-mail: lidong@pmo.ac.cn \\ 2 Max-Planck Institute for Solar System Research, 37077 Göttingen, Germany \\ ${ }^{3}$ University of Chinese Academy of Sciences, Beijing 100049, PR China
}

Received 12 January 2015 / Accepted 16 December 2015

\begin{abstract}
Flare kernels brighten simultaneously in all Solar Dynamics Observatory (SDO) Atmospheric Imaging Assembly (AIA) channels making it difficult to determine their temperature structure. The Interface Region Imaging Spectrograph (IRIS) is able to spectrally resolve Fe XXI emission from cold chromospheric brightenings, so it can be used to infer the amount of Fe XXI emission in the $131 \AA$ AIA channel. We use observations of two small solar flares seen by IRIS and SDO to compare the emission measures (EMs) deduced from the IRIS Fe XXI line and the AIA $131 \AA$ channel to determine the fraction of Fe XXI emission in flare kernels in the $131 \AA$ channel of AIA. Cotemporal and cospatial pseudo-raster AIA images are compared with the IRIS results. We use multi-Gaussian line fitting to separate the blending chromospheric emission so as to derive Fe XXI intensities and Doppler shifts in IRIS spectra. We define loop and kernel regions based on the brightness of the $131 \AA$ and $1600 \AA$ intensities. In the loop regions the Fe XXI EMs are typically $80 \%$ of the $131 \AA$ values, and range from $67 \%$ to $92 \%$. Much of the scatter is due to small misalignments, but the largest site with low Fe XXI contributions was probably affected by a recent injection of cool plasma into the loop. In flare kernels the contribution of Fe XXI increases from less than $10 \%$ at the low-intensity $131 \AA$ sites to $40-80 \%$ in the brighter kernels. Here the Fe XXI is superimposed on bright chromospheric emission and the Fe XXI line shows blueshifts, sometimes extending up to the edge of the spectral window, $200 \mathrm{~km} \mathrm{~s}^{-1}$. The AIA $131 \AA$ emission in flare loops is due to Fe XXI emission with a 10-20\% contribution from continuum, Fe XXIII, and cooler background plasma emission. In bright flare kernels up to 52\% of the $131 \AA$ is from cooler plasma. The wide range seen in the kernels is caused by significant structure in the kernels, which is seen as sharp gradients in Fe XXI EM at sites of molecular and transition region emission.
\end{abstract}

Key words. Sun: flares - Sun: UV radiation - line: profiles - techniques: spectroscopic

\section{Introduction}

The flare impulsive phase is characterized by a sudden increase in chromospheric and hard X-ray emission (Fletcher \& Hudson 2001; Fletcher et al. 2011). The emission is concentrated in small bright kernels and along ribbons, coinciding with magnetic field concentrations where rapid chromospheric heating drives hot plasma upward into the corona (Mason et al. 1986; Teriaca et al. 2006; Benz 2008; Ning \& Cao 2011). The kernels are thought to be the chromospheric signature of magnetic reconnection in the corona (Qiu et al. 2002; Fletcher et al. 2004; Gan et al. 2008). Spectroscopic observations have revealed hightemperature, high-velocity blueshift and cooler redshift emission (Antonucci et al. 1982; Milligan et al. 2006; Teriaca et al. 2006; Young et al. 2013) compatible with models of chromospheric evaporation.

Observations of the kernels show simultaneous brightening in all Atmospheric Imaging Assembly (AIA) extreme ultraviolet (EUV) channels (Brosius \& Holman 2012; Fletcher et al. 2013; Young et al. 2013). The analysis by Brosius \& Holman (2012) that compared AIA images with Coronal Diagnostic Spectrometer (CDS) spectra showed that in a small GOES B4.8 flare a significant fraction of the hot channel $94 \AA$ and $131 \AA$ emission could be attributed to the brightening of the transition region and lower coronal lines. On the other hand, Fletcher et al. (2013) attributed all the $131 \AA$ brightening in an M1.0 flare to plasma with temperatures greater than 10 MK. In an M1.1 flare, the Young et al. (2013) analysis of Extreme-Ultraviolet Imaging Spectrometer (EIS) spectra found almost equal emission measures (EMs) across the observed temperature range from 0.1 to $10 \mathrm{MK}$, but did not discuss the contributions to the AIA channel images.

The main contribution to the $131 \AA$ channel in flares is the Fe XXI $128.75 \AA$ line (O’Dwyer et al. 2010; Milligan \& McElroy 2013). The same ion, Fe XXI, produces the strong flare line at 1354.08 $\AA$ in the Interface Region Imaging Spectrograph (IRIS) spectra. Therefore, by comparing simultaneous IRIS and AIA observations it may be possible to determine the contribution of Fe XXI to the 131 channel and hence resolve the the question of the cool plasma emission contribution to the 131 channel. Using the same ion, has the advantage that the line ratios are independent of ionization and only weakly dependent on temperature.

The forbidden line of Fe XXI at $1354.08 \AA$ has been used in several spectroscopic studies to investigate hot plasma flow during flares (Doschek et al. 1975; Cheng et al. 1979; Mason et al. 1986; Feldman et al. 2000; Innes 2001; Kliem et al. 2002; Wang et al. 2003). More recently Young et al. (2015) described high spatial and spectral resolution IRIS observations of Fe XXI from hot flare kernels and loops with temperatures of about $10 \mathrm{MK}$ from an X1 class flare. Their results support the chromospheric evaporation model. The Mason et al. (1986) observations indicated that the Fe XXI line is often blended with CI. The higher resolution spectra analyzed by Young et al. (2015) show that the 


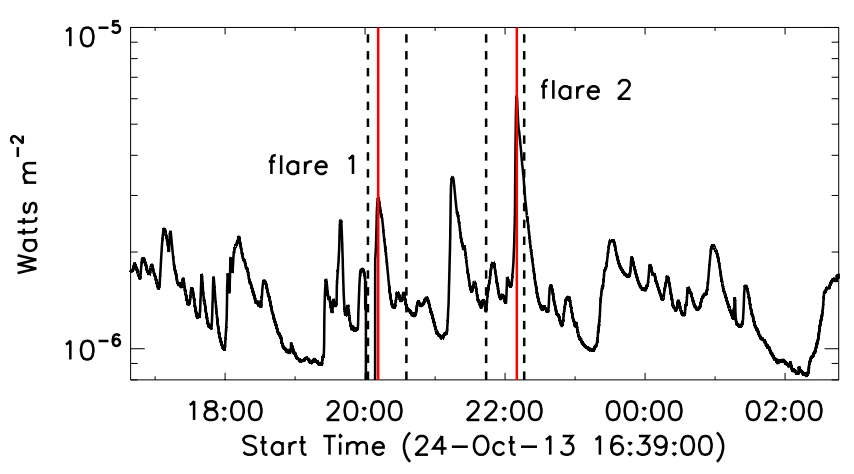

Fig. 1. GOES 1.0-8.0 A flux during the observing period. The two flares analyzed are labeled. Red lines are drawn at the times of the peak flux, while the dashed lines show the start and end times of the of IRIS raster 6 (between 20:02 UT and 20:35 UT) and raster 9 (from 21:43 UT to $22: 16 \mathrm{UT}$ ).

Fe XXI kernel emission may, in addition, be blended with other chromospheric and possibly molecular lines. Molecular hydrogen was identified at the footpoints of X-ray loops with SUMER spectra (Innes 2008). To obtain estimates of the Fe XXI EM, the strength of these chromospheric lines needs to be taken into account. For the analysis in this paper, we investigated a number of flare kernel spectra and developed an algorithm for simultaneously fitting the Fe XXI, blending lines, and continuum.

The EM obtained from the IRIS $1354 \AA$ line was compared with that derived from simultaneous Solar Dynamics Observatory (SDO) AIA $131 \AA$ images to determine the contribution of cooler line emission to the $131 \AA$ channel at the site of flare kernels. We find that the Fe XXI observed by IRIS can account for about $80 \%$ of the SDO/AIA $131 \AA$ emission in flare loops and $40-80 \%$ of the emission in flare kernels. Assuming that an additional $20 \%$ of the Fe XXI is continuum (Milligan \& McElroy 2013), we find that up to $52 \%$ of the $131 \AA$ channel emission is from cooler plasma in the flare kernels.

\section{Observations}

The observed active region, AR 11875, produced four C-class flares during the period 16:39 UT on 24 October 2013 and 02:46 UT on 25 October 2013. We obtained high-quality cotemporal and cospatial IRIS and AIA observations for flares at 20:10 and 22:05 UT (Fig. 1). During the early part of the sequence SDO was off-pointing, and during the 21:09 UT flare the IRIS spectra were badly affected by a large number of particle hits. Fig. 2 shows the AIA $131 \AA$ and corresponding slit-jaw (SJ) $1400 \AA$ images of the two flares analyzed. The GOES fluxes of the two flares are shown in Fig. 1. Flare 1 peaked at about 20:10 UT and ended at about 20:22 UT, while flare 2 peaked at about 22:10 UT and stopped at about 22:15 UT.

\subsection{AlA observations}

In this analysis, AIA images (Lemen et al. 2012) from the 131 and $1600 \AA$ channels are used for comparison with the IRIS data. The AIA level 1.0 data were downloaded and processed to level 1.5 using the standard solarsoft (SSW) routines. Then using the code drot_map.pro in SSW, the active region with a field of view of $420^{\prime \prime} \times 420^{\prime \prime}$ are selected from the AIA full disk images (see Fig. 2). The images from different AIA filters have alignment uncertainties of about 1-2 pixels
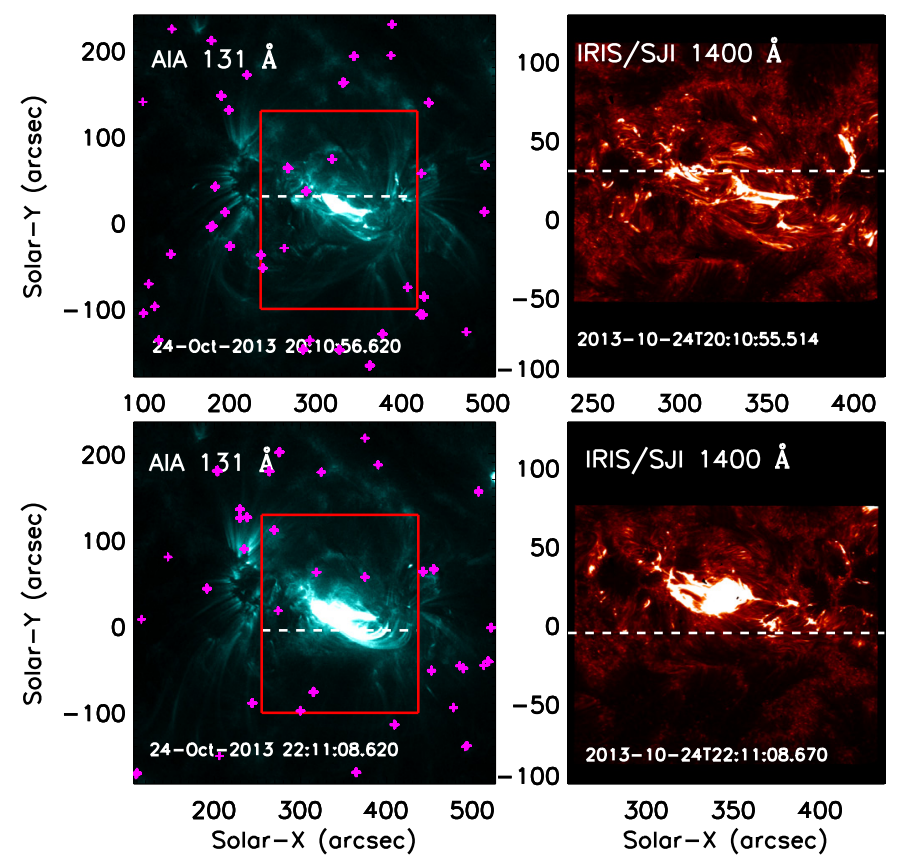

Fig. 2. Left: AIA $131 \AA$ images of the active region at the times of the two flares. The red boxes are the positions of the IRIS SJ images. Right: IRIS SJ $1400 \AA$ images of the two flares analyzed. The position of the spectrometer slit at the start of the flare is seen as a white dashed horizontal line. The purple +'s indicate the re-spike pixels in the AIA $131 \AA$ images with the code "aia_respike".

(e.g., Young et al. 2013). Therefore the AIA $131 \AA$ images are slightly shifted (i.e., $0.5-2$ pixels) with respect to the $1600 \AA$ images because we obtained the best alignment of the $1600 \AA$ with the IRIS $1400 \AA$ and the $131 \AA$ with the IRIS Fe XXI.

The downloaded AIA data are already de-spiked, which often removes flare kernel emission and these can be put back with the aia_respike routine (see Young et al. 2013). In our study, we compared the "re-spike" data and the level 1 data at $131 \AA$ as shown with the purple plus signs in Fig. 2. This shows that the re-spiked pixels are far away from the IRIS slit position, and they are not within the regions of our study. This was the case for all other $131 \AA$ images. Furthermore, during big flares the AIA $131 \AA$ channel is often saturated. The two flares in this paper are quite small and very few pixels in the $131 \AA$ channel were saturated. Fortunately, those saturated pixels are far away from the IRIS raster slit. In other words, neither the de-spiking nor the saturated pixels are a problem in our study.

\subsection{IRIS observations}

IRIS is a NASA Small Explorer Mission launched in June 2013, and its main science is an investigation of the dynamics of the Sun's chromosphere and transition region (McIntosh et al. 2013). As described in detail by De Pontieu et al. (2014), IRIS is a high-resolution spectrograph and slit-jaw (SJ) camera that obtains spatial resolution of $0.33-0.4$ arcsec and spectral resolution of $\sim 26 \mathrm{~m} \AA$ (or $\sim 52 \mathrm{~m} \AA$ in second order). There are three IRIS wavelength bands: (i) $1332-1358 \AA$, which includes the strong C II doublet, Fe XXI, and a number of strong C I lines; (ii) 1389-1406 A, which includes the Si IV doublet and density sensitive O IV lines; and (iii) 2782-2834 Å, which includes Mg II h and $\mathrm{k}$ lines. Here we analyze lines in the 1332-1358 $\AA$ range. 

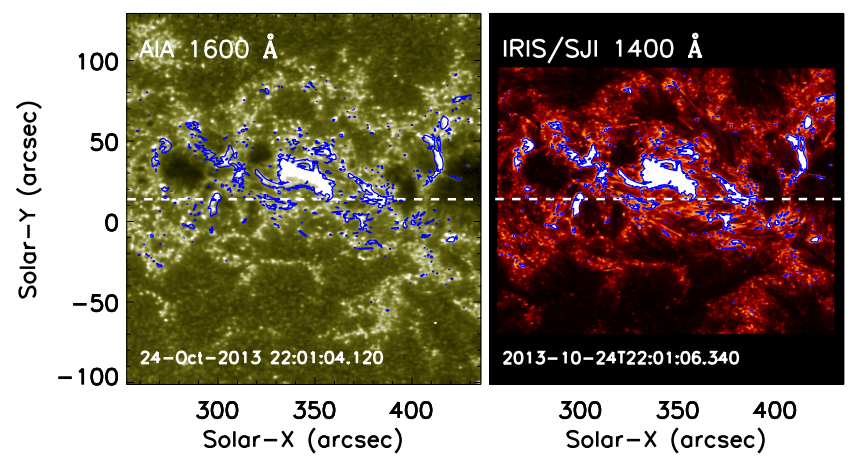

Fig. 3. Coalignment of the SDO and IRIS images. Left: the AIA image at $1600 \AA$. Right: the SJ image at $1400 \AA$. The white dashed line is the IRIS slit position, and the blue contours are the SJ intensity at the level of $800 \mathrm{DN}$.

IRIS also obtains SJ images centered at 1330, 1400, 2796, and $2832 \AA$.

The observations described here were designed with the aim of capturing the structure and spectra of flares by making rapid rasters across a flaring active region for as long a period as telemetry constraints allowed. The IRIS rasters were obtained by taking $641.01^{\prime \prime}$ steps across a region $174^{\prime \prime} \times 63^{\prime \prime}$ with the roll angle $90^{\circ}$ (i.e., the slit was oriented E-W). Simultaneous SJ images at $1400 \AA$ with a field of view $174^{\prime \prime} \times 166^{\prime \prime}$ and cadence $32 \mathrm{~s}$ were also obtained and used mainly for coalignment with $1600 \AA$ AIA images. Because continuum emission from the temperature minimum is dominant in many of the bright features seen in both sets of images, coalignment is possible to within the AIA pixel size, 0.6" (Fig. 3).

The step cadence was $31.6 \mathrm{~s}$ and the exposure time $30 \mathrm{~s}$, thus the raster cadence was $33 \mathrm{~m} 42 \mathrm{~s}$. Eighteen rasters were obtained in the roughly ten-hour period. The pixel size along the slit was at the highest resolution $0.167^{\prime \prime}$, but four times spectral binning and a restricted number of spectral windows were obtained to save telemetry. In this case we used the flare list of lines, which consisted of the C II, 1343, Fe XII, O I, and Si IV far-ultraviolet (FUV) windows. Here we only discuss spectra from windows in the short wavelength FUV band. The spectral resolution was 4*12.72 $\mathrm{m} \AA /$ pixel, equivalent to $10.88 \mathrm{~km} \mathrm{~s}^{-1} /$ pixel.

IRIS level 2 data were downloaded. These data were calibrated and corrected for image distortions. Several of the images were badly affected by particle hits and hot or dead pixels, which were removed using a despiking routine that detected persistent hot/dead pixels and sudden changes in intensity since they can seriously distort the line fitting results. Care is taken to ensure that only isolated bright pixels or small pixel groups are removed, and to leave intensity changes due to sudden line broadening. Figure 4 shows the effect of de-spiking. In addition, small spectral shifts caused by thermal drifts and the spacecraft orbital velocity were corrected with the routine iris_orbitvar_corr_12.pro (McIntosh et al. 2013; Tian et al. 2014; Cheng et al. 2015$)$ in the SSW package.

\subsection{Flare ribbon spectra}

The ribbons are characterized by many narrow, bright emission lines. The Fe XXI emission blends with both known and unknown lines from neutral and singly ionized species, as well as molecular fluorescence lines. To determine the Fe XXI intensity we need to extract the blending chromospheric emission.

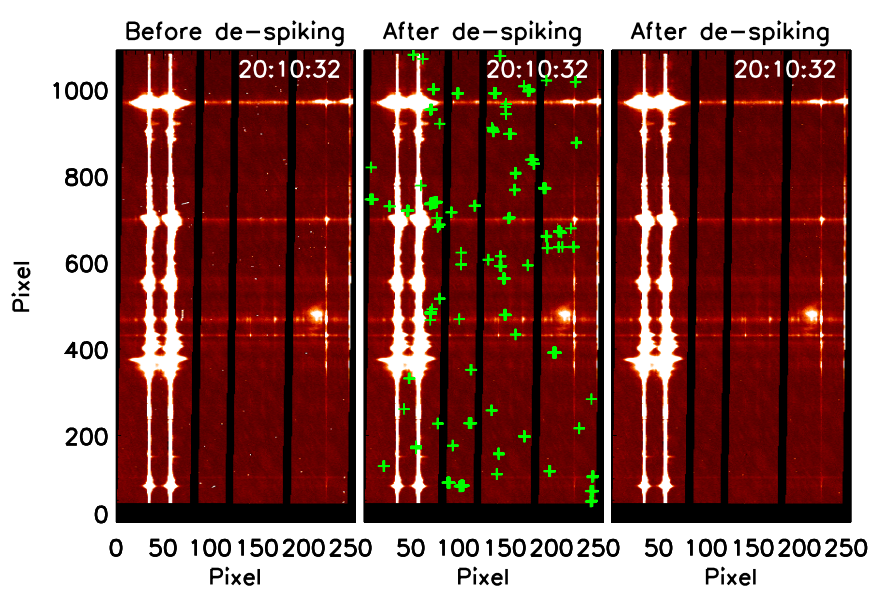

Fig. 4. IRIS raster images before (left) and after de-spiking (right). The middle panel is an image after de-spiking with the bad pixels marked with green + 's.
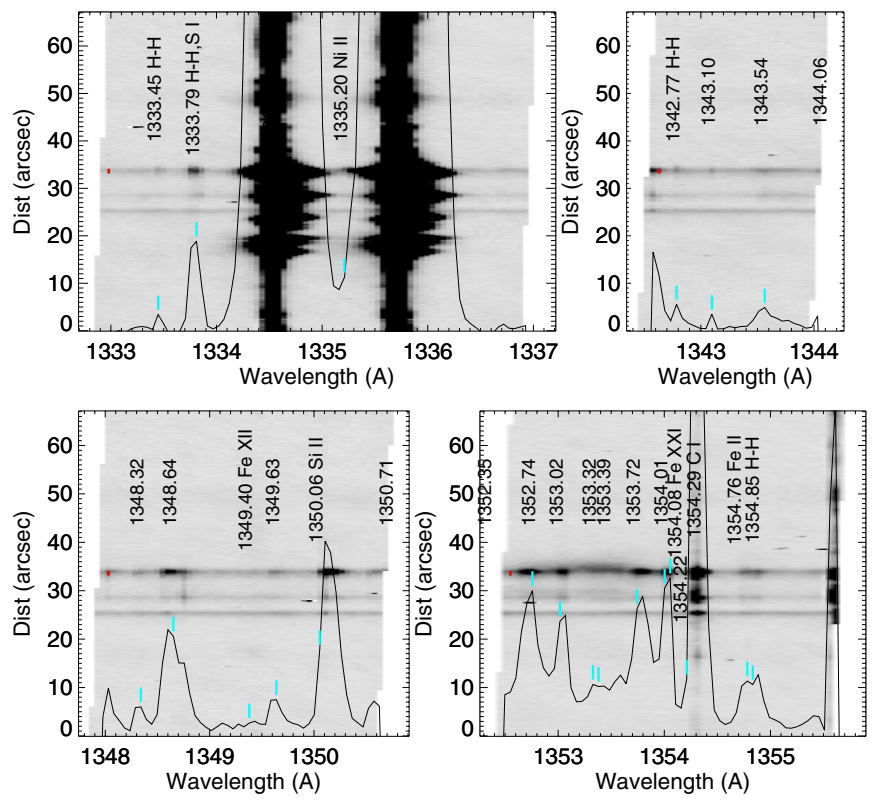

Fig. 5. Flare footpoint spectra taken on 24 October 2013. The overplotted line spectra are the average across the small bright region marked by a red line on the left-hand side of each image. The main lines are labeled and indicated by light blue vertical ticks just above the line spectra.

We have identified the main blending lines by looking at kernel spectra from other IRIS datasets at higher spectral resolution, as well as many spectra in this dataset to find out which other observed lines behave similarly to the chromospheric lines in the Fe XXI window. An example of a flare footpoint spectrum taken with this sequence is shown in Fig. 5. For comparison, the chromospheric lines seen during a C1.5 flare from AR11861 (-133", $-285^{\prime \prime}$ ) at 00:46 UT on 12 October 2013, taken with the full spectra resolution, are shown in Fig. 6.

The main chromospheric lines in the O I window, apart from the C I 1354.29 $\AA$ line, are the Fe II lines at 1353.02, 1354.01, and $1354.76 \AA$; the Si II lines at 1352.74 and $1353.72 \AA$; and the unidentified lines at 1353.32 and $1353.39 \AA$. Actually, other papers (e.g., Graham \& Cauzzi 2015; Li et al. 2015; Tian et al. 2015) have attempted multi-Gaussian fits to the Fe XXI line from IRIS spectral observations. In this paper, to obtain the Fe XXI intensities, we fixed or constrained the positions and 

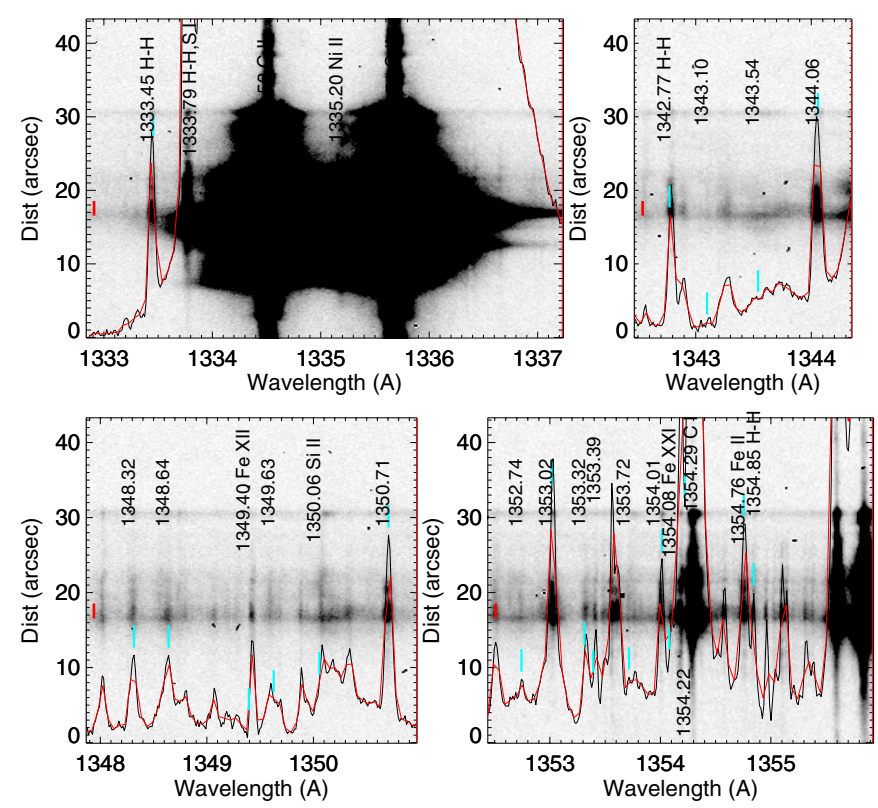

Fig. 6. Flare footpoint spectra taken on 12 October 2013 at full resolution. The overplotted line spectra are the average across the small bright region marked by a red line on the left-hand side of each image and, in red, the same spectra binned by a factor of 4 , which is the resolution used on 24 October 2013.

widths of these lines and set their intensities to have a specified ratio to well-resolved lines from similar species. In total we fit 17 Gaussian lines superimposed on a linear background fitted across the wavelength region (i.e., 1333.01-1355.55 ^) covered by the four IRIS spectral windows. Table 1 lists the lines used in the fitting procedure. Lines with fixed positions are indicated by a superscript " 1 ". The two Si II lines, indicated with a superscript "2", are constrained to match the shift of the unblended Si II at $1350.06 \AA$. The widths are fixed or constrained as given in column 4. For example, the Si II 1353.72 line has a maximum width of $260 \mathrm{~m} \AA$, while the line width of Fe II 1353.02 is fixed at $41 \mathrm{~m} \AA$. The peak intensities of the blending lines are forced to be in a fixed ratio (Col. 6) with the lines that they are tied to (Col. 5). For example, the peak intensity of the blending line Si II 1353.72 is tied to the emission line Si II 1350.06 in the Fe XII window, and the intensity ratio is 0.49 . The positions and intensity ratios of the lines were determined by fitting Gaussians to all the narrow (identified and unidentified) lines in 17 spectra in the dataset with bright molecular and chromospheric lines but no Fe XXI emission. For each line, the median position from the 17 spectral fits was taken as the line's fixed position. Then for each narrow line blending with the Fe XXI, we calculated its intensity ratio with respect to all unblended narrow lines. We used the variation in the intensity ratios to identify which line each blended lines should be tied to, and the median of the ratio was used to determine the strength of the blended line from the intensity of its tied line. The lines used in the final fitting procedure to obtain the Fe XXI intensities are marked and the regions of background are indicated by the red dashed lines in Fig. 7. At most positions the fits appear to be successful.

\section{Emission measures of Fe XXI}

The observed intensity is given by

$I=E_{\mathrm{f}} \int G\left(T, n_{\mathrm{e}}\right) n_{\mathrm{e}}^{2} \mathrm{~d} z$

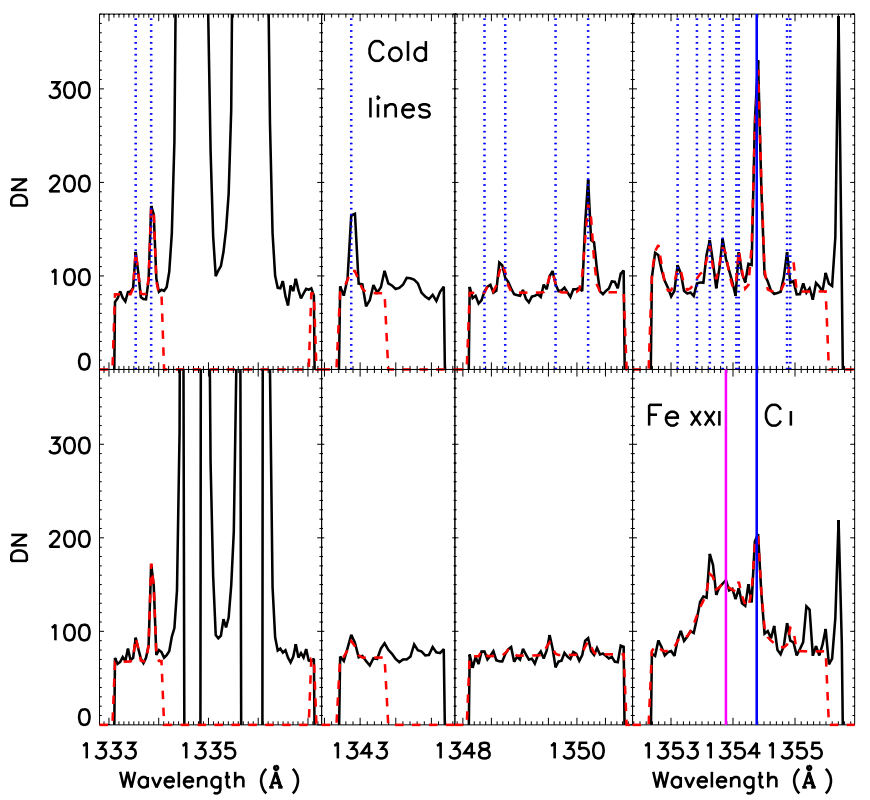

Fig. 7. Spectra (black) and multi-Gaussian fits (red). The blue dashed lines mark the positions of cold lines, the blue solid line marks the C I line, while the purple line marks the Fe XXI line.

Table 1. Seventeen emission lines from the four IRIS spectral windows used in the multi-Gaussian fit.

\begin{tabular}{|c|c|c|c|c|c|}
\hline IRIS window & $\begin{array}{l}\text { Wavelength } \\
(\AA)\end{array}$ & Ion & $\begin{array}{l}\text { Width } \\
(\mathrm{m} \AA)\end{array}$ & $\begin{array}{l}\text { Intensity } \\
\text { tied to }\end{array}$ & Ratio \\
\hline & $1333.45^{1}$ & $\mathrm{H}_{2}$ & 31 & - & - \\
\hline $\mathrm{C}$ II & $1333.79^{1}$ & $\mathrm{H}_{2}, \mathrm{~S} \mathrm{I}$ & 41 & - & - \\
\hline 1343 & $1342.77^{1}$ & $\mathrm{H}_{2}$ & 31 & - & - \\
\hline \multirow{4}{*}{ Fe XII } & $1348.32^{1}$ & - & 41 & - & - \\
\hline & $1348.64^{1}$ & - & 31 & - & - \\
\hline & $1349.63^{1}$ & - & 41 & - & - \\
\hline & 1350.06 & Si II & $\leq 260$ & - & - \\
\hline \multirow{10}{*}{ O I } & $1352.74^{2}$ & Si II & $\leq 260$ & Si II 1350.06 & 0.54 \\
\hline & $1353.02^{1}$ & Fe II & 41 & Fe II 1354.76 & 1.85 \\
\hline & $1353.32^{1}$ & - & 88 & $\mathrm{H}_{2} 1342.77$ & 0.79 \\
\hline & $1353.39^{1}$ & - & 31 & $\mathrm{H}_{2} 1342.77$ & 1.50 \\
\hline & $1353.72^{2}$ & Si II & $\leq 260$ & Si II 1350.06 & 0.49 \\
\hline & $1354.01^{1}$ & Fe II & 41 & Fe II 1354.76 & 3.43 \\
\hline & 1354.08 & Fe XXI & $\geq 230$ & - & - \\
\hline & 1354.29 & C I & $\leq 130$ & - & - \\
\hline & $1354.76^{1}$ & Fe II & 42 & - & - \\
\hline & $1354.85^{1}$ & $\mathrm{H}_{2}$ & 31 & $\mathrm{H}_{2} 1342.77$ & 2.0 \\
\hline
\end{tabular}

Notes. 1: The emission lines with fixed positions in the multi-Gaussian fit. 2: The emission lines with constrained positions in the multiGaussian fit.

where $n_{\mathrm{e}}$ is the electron density, $T$ is electron temperature, $z$ is the line-of-sight coordinate, $G\left(T, n_{\mathrm{e}}\right)$ is the contribution function of the line including the atomic abundance, and $E_{f}$ is the instrument effective area or response function. In the equation above the units of $I$ depend on the units of the $E_{f}$. As discussed below, IRIS is a spectrometer and the intensities are given in $\mathrm{DN} \mathrm{s}{ }^{-1}$; AIA is an imager and the intensity unit is $\mathrm{DN} \mathrm{px}^{-1} \mathrm{~s}^{-1}$. To convert intensity to emission measure, we need to divide by the respective response functions in the appropriate units. Since the contribution functions of the IRIS and AIA Fe XXI lines are sharply peaked in temperature and are formed over the same heights, the contribution functions can be removed from the 
D. Li et al.: Observations of solar flares with IRIS and SDO

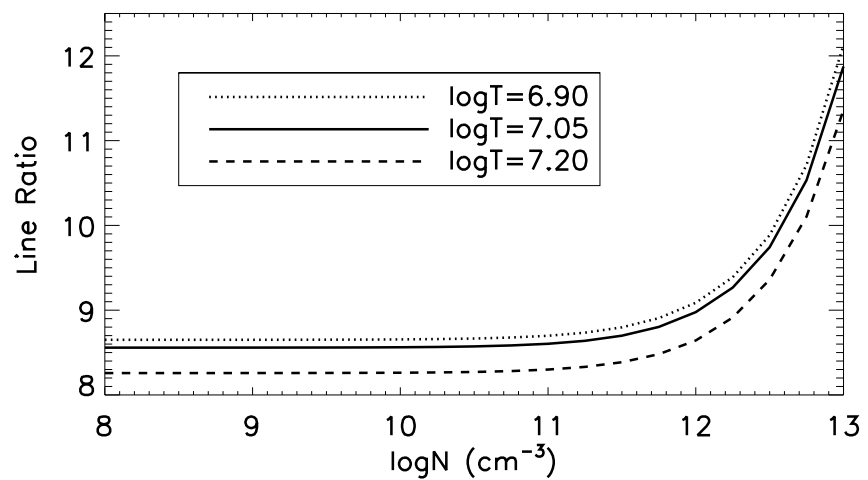

Fig. 8. Variation of the Fe XXI 128.75/1354.08 ratio as a function of density, which is from CHIANTI V. 7.1.3 (Landi et al. 2013).

integral. The emission measure in the Fe XXI plasma can then be calculated from

$$
E M=\int n_{\mathrm{e}}^{2} \mathrm{~d} z=I /\left(E_{\mathrm{f}} G\left(T_{\text {peak }}\right)\right) \quad \mathrm{cm}^{-5}
$$

The temperature response, $E_{\mathrm{f}} G\left(T_{\text {peak }}\right)$, for the AIA $131 \AA$ channel and the IRIS $1354 \AA$ line are shown in Fig. 9. The AIA function has been computed with the procedure aia_get_response.pro with the calibration appropriate to the time of the observation at the default pressure in CHANTI of $10^{15} \mathrm{~cm}^{-3} \mathrm{~K}$ (Boerner et al. 2012). The keyword "chiantifix" is also set to account for emission that is not included in the CHIANTI database (Testa et al. 2012; DeRosa \& Slater 2013). The response of AIA to high- and low-temperature flare emission is believed to be accurate to $25 \%$ with these empirical corrections (Boerner et al. 2012). There are two peaks, one due to Fe VIII formed around $6 \times 10^{5} \mathrm{~K}$ and the other due to Fe XXI formed around $10^{7} \mathrm{~K}$. The main variation in the contribution function around $10 \mathrm{MK}$ comes from the dependence of ionization on temperature. The IRIS Fe XXI line is a fine structure transition within the ground state so its excitation rate is independent of temperature, but at high densities $\left(n_{\mathrm{e}}>10^{12} \mathrm{~cm}^{-3}\right)$ it suffers from collisional deexcitation. Figure 8 shows the variation of the $128.75 / 1354.08$ line ratio, computed with CHIANTI (Landi et al. 2013), as a function of density for three temperatures close to the peak of the contribution function. The temperature variation at low densities varies by less than $7 \%$ over the range shown. Only at temperatures below $4 \mathrm{MK}$ is there a significant temperature dependence on the ratio. We assumed the low-density limit and temperature $11 \mathrm{MK}$ and computed the Fe XXI response function as the line emissivity divided by the radiometric conversion coefficient, 2960 (De Pontieu et al. 2014), and the spectral scale $(0.0128 \AA)$. Again, the electron pressure was set to $10^{15} \mathrm{~cm}^{-3} \mathrm{~K}$. The radiometric conversion for the IRIS spectra is based on the International Ultraviolet Explorer (IUE) spectral radiances, which has an uncertainty of $10-15 \%$ (one $\sigma$ ). Both the AIA and IRIS emissivities are computed at the same pressure, elemental abundance, and ionization. In the analysis of the Fe XXI contribution to the 131 channel, we assume that the ratio of the intensities is constant.

Fe XXI emission measure maps can be obtained by dividing the observed intensities (DN s${ }^{-1}$ ) by the value of the peak response, at $11 \mathrm{MK}$, of the respective functions. To compare the IRIS and AIA results pixel-by-pixel pseudo rasters were constructed from a series of AIA images by selecting the overlapping AIA pixels from the closest in time AIA image for each IRIS raster position. Along the slit, each AIA pixel $\left(0.6^{\prime \prime}\right)$ is

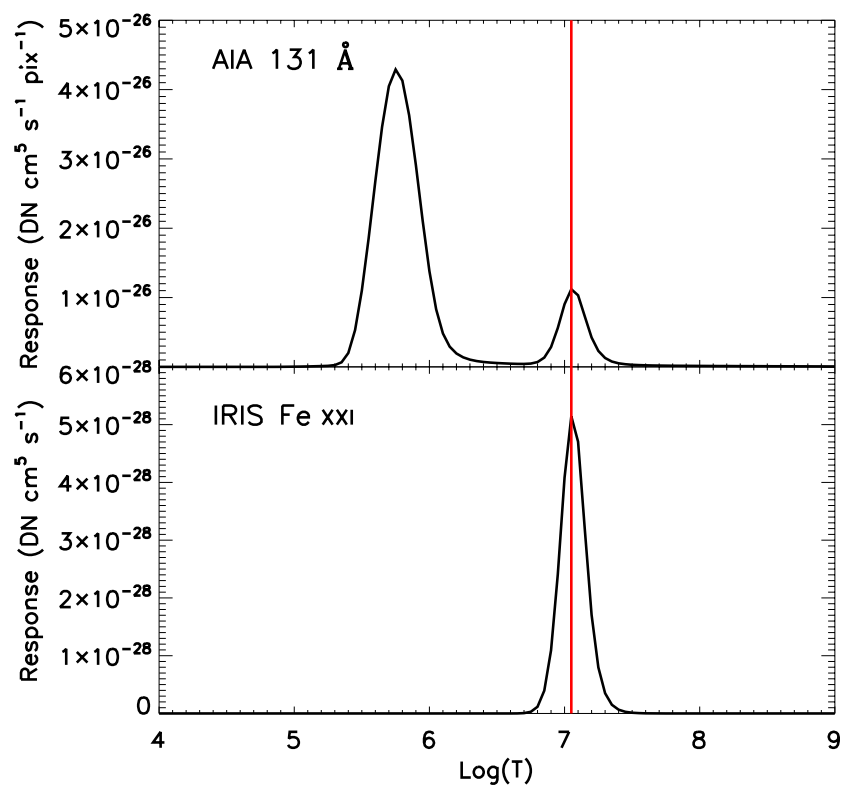

Fig. 9. Temperature response of the AIA $131 \AA$ channel (top) and the IRIS Fe XXI $1354 \AA$ line (bottom). The red lines are drawn at the peak of the response curves, $11 \mathrm{MK}$.

about 3.6 IRIS pixels $\left(0.167^{\prime \prime}\right)$. We map the IRIS pixels onto the AIA grid and average the IRIS intensity in each AIA pixel. The IRIS raster step size (1.01") is larger than the AIA pixels so in the raster direction the single overlapping IRIS pixel is used. Thus, the IRIS data have been averaged across $\sim 3.6$ pixels in the slit direction so that the IRIS data are re-binned to the scale of the AIA data. In the raster direction, the AIA pseudo rasters are taken from the closest time AIA data.

\section{Results}

\subsection{Images and spectra}

There were two well-observed solar flares, as shown in Fig. 2. The IRIS spectra of the two flares were fitted, as described, with 17 Gaussians superimposed on a linear background. The primary lines of interest are the Fe XXI 1354.08 $\AA$ and, to a lesser extent, C I $1354.29 \AA$ A. Figs. 10 and 12 compare IRIS Fe XXI and CI intensity images with AIA 131 and $1600 \AA$ pseudo-rasters of flare 1 and flare 2 . The regions of enhanced C I identify the flare kernels. The Fe XXI Doppler velocity is shown in the bottom left panel. From the velocity images we can see that the Fe XXI is blueshifted near the start of the flare near the flare kernels. As shown, bright C I overlaps very well with the bright $1600 \AA$ emission. The highest velocity measured is about $200 \mathrm{~km} \mathrm{~s}^{-1}$.

To look closely at the emission from the kernels, we extracted spectra from almost neighboring positions along the slit. The points are shown by plus signs ("+") in Figs. 10 and 12. In Figs. 11 (flare 1) and 13 (flare 2), we show spectral images and spectra from the first raster step with Fe XXI kernel emission, as well as spectra taken at the same positions from the earlier and later raster steps. The resultant fits appear to be very good. In both flares, the Fe XXI appears as a broad line below the narrow chromospheric lines. The main sources of uncertainty in the Fe XXI intensity come from the background fit, the edge of the spectral window, and the possibly non-Gaussian Fe XXI profile. The background is fitted across all spectral windows and so is fairly well constrained, and the window edge only affects the few positions where Fe XXI is blueshifted by more than $150 \mathrm{~km} \mathrm{~s}^{-1}$. 


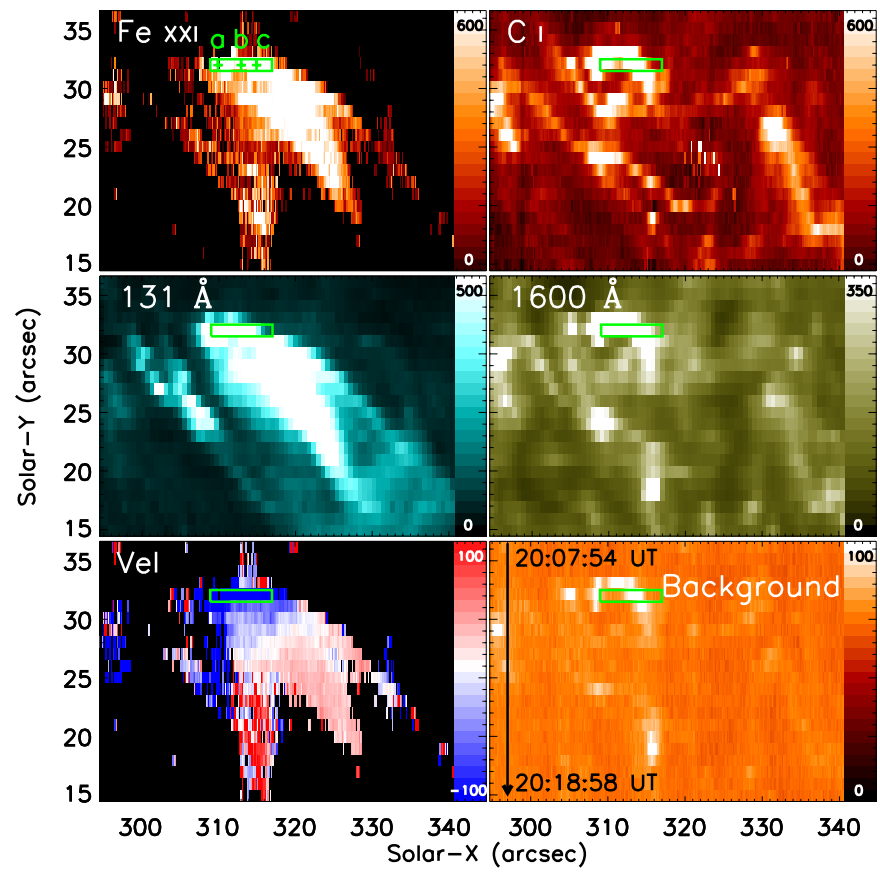

Fig. 10. Images of the first flare: (top) IRIS Fe XXI $1354.08 \AA$ and C I 1354.29 Å intensity; (middle) AIA $131 \AA$ and $1600 \AA$ pseudo-raster intensity; (bottom) IRIS Fe XXI Doppler velocity and continuum intensity. The green rectangle marked with +'s indicates the region and sites of the spectra shown in Fig. 11. The arrow indicates the time direction; the duration of these images is $11 \mathrm{~m} 4 \mathrm{~s}$. The duration of the raster is indicated in Fig. 1.

As well as assessing the fits by eye we also computed the $\chi^{2}$ for the spectral region around the Fe XXI line. The values were generally less than one. At one or two positions, the $\chi^{2}$ was large $(>10)$. Here the peak of the C I line could not be fit correctly by a Gaussian possibly due to optical depth effects. Occasionally individual narrow lines blending with Fe XXI caused $\chi^{2}$ of about 3 at the position of the Fe XXI line. Since they were much narrower than the Fe XXI they did not affect the Fe XXI intensity.

\subsection{Emission measures of AIA $131 \AA$ and Fe XXI}

From Eq. (2) we can estimate the EMs of the AIA 131 and IRIS Fe XXI emitting plasma. Figure 14 shows the result for the first flare. The upper panels are the images of the EMs with contours of the IRIS Fe XXI EM. The images of the EMs have a logarithmic scaling, and show good qualitative agreement. The lower panel shows the comparison of the two EMs. To look closer at the overlap, we selected the smaller region (surrounded by the white box) and perform a row-by-row comparison of the EMs of AIA 131 and Fe XXI in the panel underneath. The structures match closely. The AIA 131 EMs are more diffuse and greater than Fe XXI EMs except around positions 1 and 2. As a check on the Gaussian line fit, we show the spectra and fits at the numbered positions in Fig. 15. All fits appear to be very good and there is no special feature of position 1 or 2 to indicate why the computed Fe XXI EMs are higher than the 131 EMs.

We checked whether the higher EMs were due to a temporal mismatch between IRIS and AIA. AIA produced two $131 \AA$ exposures during each IRIS exposure (30 s) so we compared with both of the overlapping AIA images separately for these and all other points and found only very small differences. We therefore
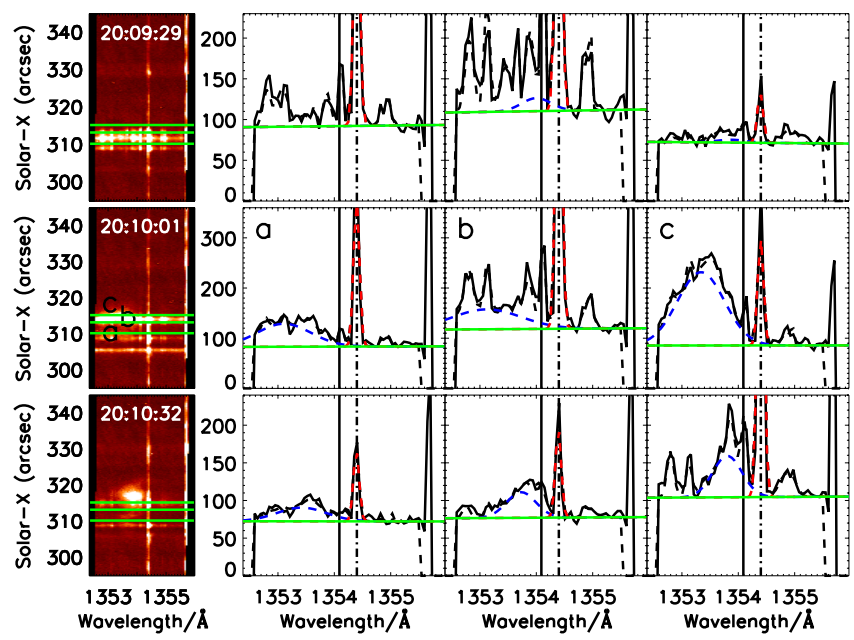

Fig. 11. Flare-kernel spectra from the region inside the green rectangle in Fig. 10: (left-middle) the spectrogram of the region marked with a green rectangle in Fig. 10, with before (left-top) and after (left-bottom) spectrograms. The right panels show the spectra (solid) and their fits (dashed) at the positions indicated by the green lines in the left panel. The vertical solid lines give the rest wavelength of Fe XXI and the vertical dashed-dot lines give the rest wavelength of $\mathrm{C}$ I line. The green solid line is the background, the blue dashed line is the background + Fe XXI line fit, and the red dashed line is the background + C I line fit.

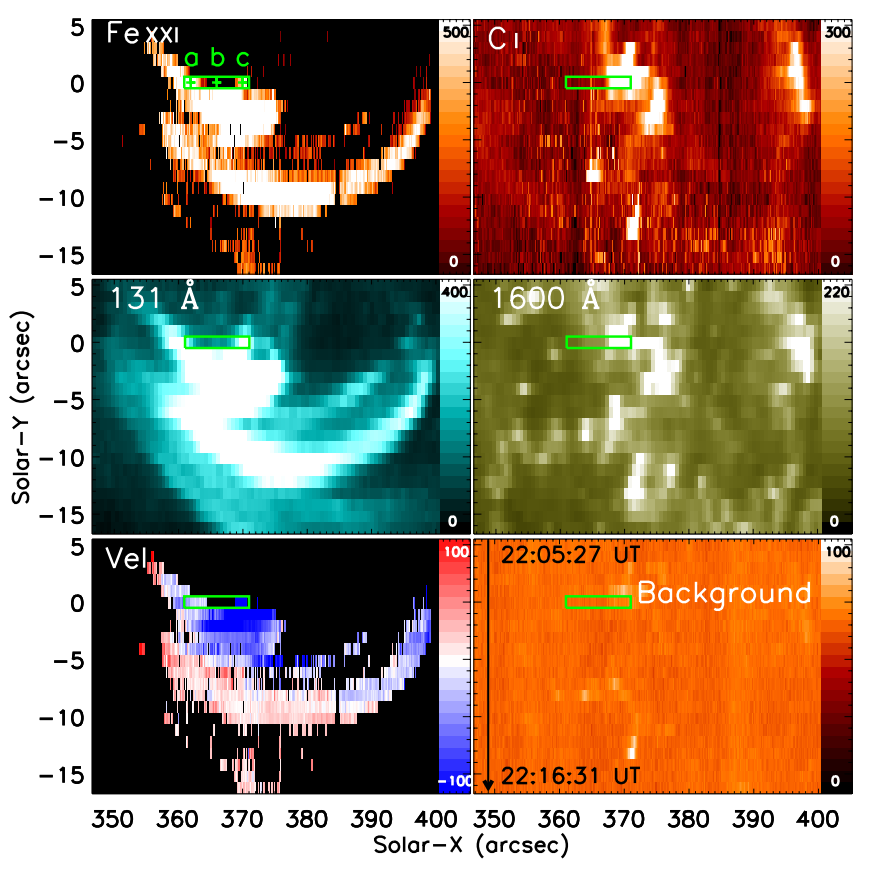

Fig. 12. Images of the second flare. (top) IRIS Fe XXI $1354.08 \AA$ and C I 1354.29 Å intensity; (middle) AIA $131 \AA$ A and $1600 \AA$ pseudo-raster intensity; (bottom) IRIS Fe XXI Doppler velocity and continuum intensity. The green rectangle marked with +'s indicates the region and sites of the spectra shown in Fig. 13. The arrow indicates the time direction; the duration of these images is $11 \mathrm{~m} 4 \mathrm{~s}$. The duration of the raster is indicated in Fig. 1.

conclude that it is not a temporal effect. It could be an effect of the two different spatial resolutions. The IRIS slit only samples about half of the AIA pixel so if the features are very concentrated, the filling factor may be less in AIA than in IRIS. This would result in a lower AIA than IRIS EM. 

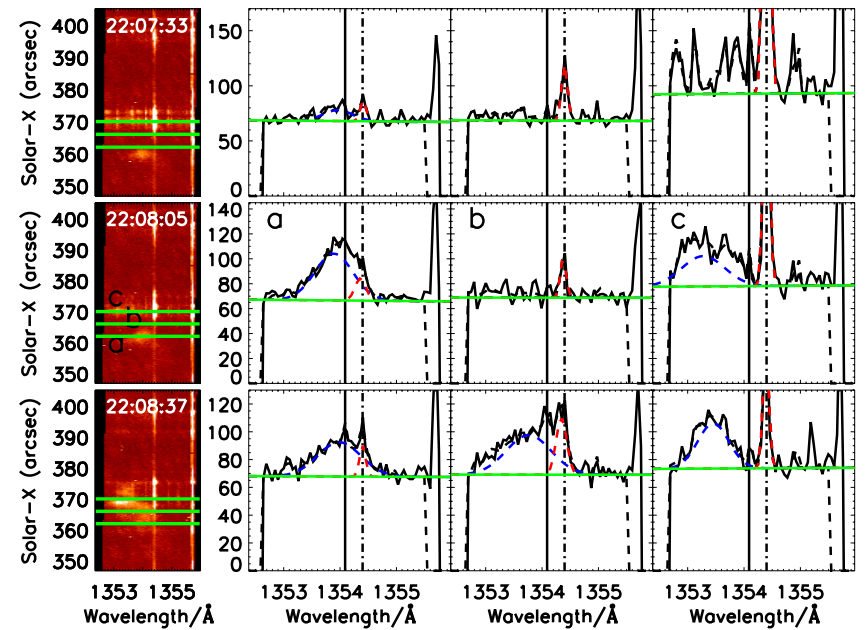

Fig. 13. Flare-kernel spectra from the region inside the green rectangle in Fig. 12: (left-middle) the spectrogram at the time of the rectangle region in Fig. 12, with before (top) and after (bottom) spectrograms. Right: spectra and their fits (dashed lines) at the positions marked by the green lines in the left panel. The vertical solid lines indicate the rest wavelength of FeXXI, and the vertical dash-dotted lines indicate the rest wavelength of $\mathrm{C}$. The green solid line is the background, the blue dashed line is the background + Fe XXI line fit, and the red dashed line is the background $+\mathrm{CI}$ line fit.
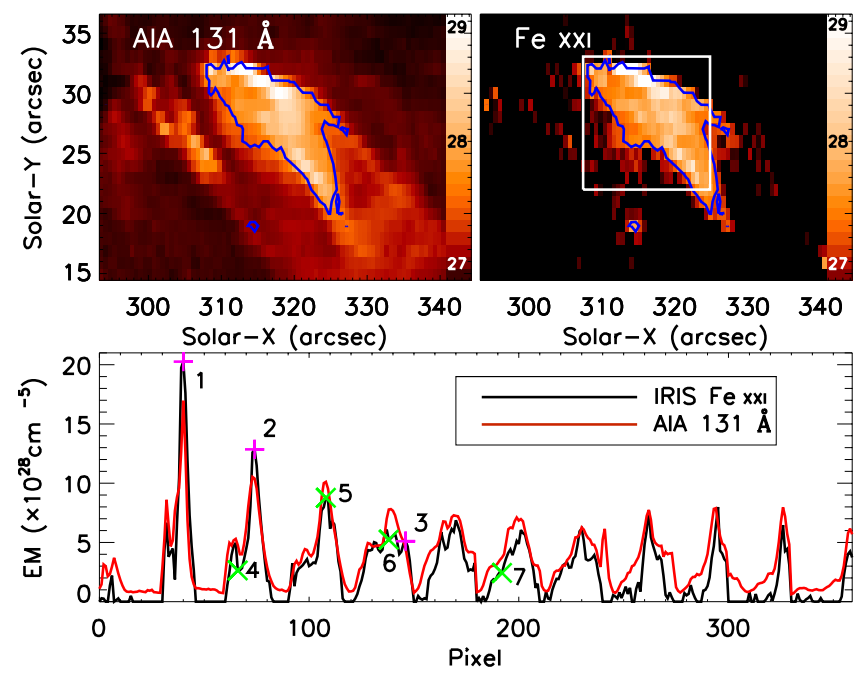

Fig. 14. Upper: emission measures computed from AIA $131 \AA$ and IRIS Fe XXI at $11 \mathrm{MK}$ for the first flare. The white box is the region over which we compare EMs in the panels below. Bottom: pixel-by-pixel and row-by-row EMs across the white box region, starting from the upper left. The black line is the Fe XXI EM, and the red line is the $131 \AA$ EM. The +'s indicate those pixels where the Fe XXI emission is higher, and the $\times$ 's mark the selected pixels shown in Fig. 15 .

Similar plots for the second flare are shown in Figs. 16 and 17. The region of bright Fe XXI is slightly larger in the second flare so we split the region into two, labeled "R1" and "R2" in Fig. 16. Again, selected spectra are shown (see Fig. 17). The overall behavior is similar to that seen in the first flare: the 131 EMs are higher and more diffuse than the Fe XXI EMs. At position 4, the IRIS EM is larger than the 131. As shown in Fig. 17, this point is also on the edge of a loop, so it may again be indicating a slight coalignment mismatch. We also note that at position 2, which is near the peak in the 131 emission, the IRIS EM is only about two-thirds of the 131 EM. As seen in Fig. 17,

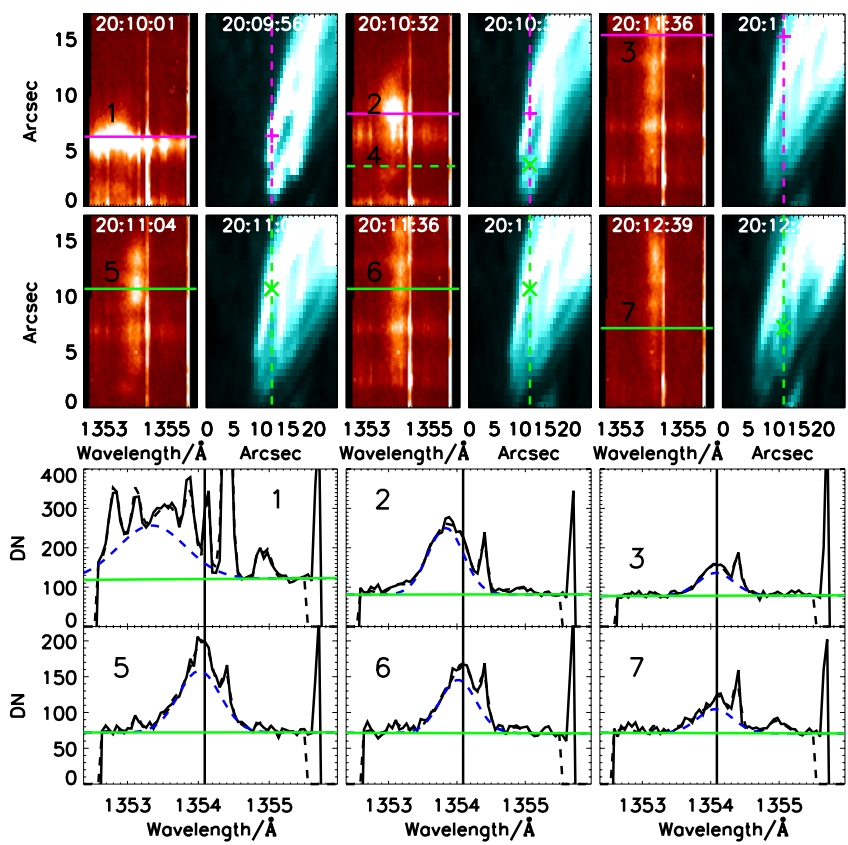

Fig. 15. Selected IRIS spectra and AIA intensities for the first flare. The vertical dashed lines in the AIA images are the positions of the IRIS slits. The numbers on the IRIS images show the positions of the selected points in Fig. 14. The +'s and X's in the AIA images indicate the positions of these points in the AIA images. Underneath are the IRIS spectra (solid black line) and their fits (black dashed line) for the selected positions. The green solid line is the background, the blue dashed line is background + Fe XXI line, while the vertical line is the rest wavelengths of the Fe XXI line.

the IRIS spectra at this position show a blueshift of about $0.5 \AA$ or $100 \mathrm{~km} \mathrm{~s}^{-1}$. Blueshifts in flares are usually linked to chromospheric evaporation, which implies that a range of ionization states can be expected, including Fe VIII at this position. Further support for this conclusion comes from the time evolution that showed brightening at this site in all AIA channels at the time of the IRIS observations.

To obtain quantitative values we separate the two flare regions into loops and kernels and compare the IRIS-to-AIA EM ratios as a function of Fe XXI or 131 intensity. We plot the ratio of $131 \mathrm{EM}$ to the sum of 131 and Fe XXI EMs to avoid going off the scale when the Fe XXI EM is very low. Thus, when the $131 \mathrm{EM}$ is due to Fe XXI alone, the ratio is 0.5 . A contribution to the 131 EM from cooler lines, continuum, or Fe XXIII would produce a value closer to one, and where there is no Fe XXI contribution, the value is one. The results for the two flares are shown in Figs. 18 and 19. The selection of loop and kernel regions is based on the AIA 131 and $1600 \AA$ intensities. Pixels with $131 \AA$ intensities greater than twice $131 \AA$ average and $1600 \AA$ intensities less than twice $1600 \AA$ average are selected as loops and those with $1600 \AA$ intensities greater than twice $1600 \AA$ average are selected as kernels.

For loops the average value of the ratio is 0.54 in flare 1 and 0.55 in flare 2 . The ratio is almost constant across the intensity range. This implies that typically about $80-85 \%$ of the $131 \AA$ loop emission is from Fe XXI which is consistent with Milligan $\&$ McElroy (2013) who estimate a $20 \%$ continuum contribution to the $131 \AA$ channel during flares.

For flare 1 , the ratio ranges from 0.2 to 0.6 . The low values can be attributed to alignment or filling factor issues. For example the single point in the highest intensity bin in flare 1 is from a 

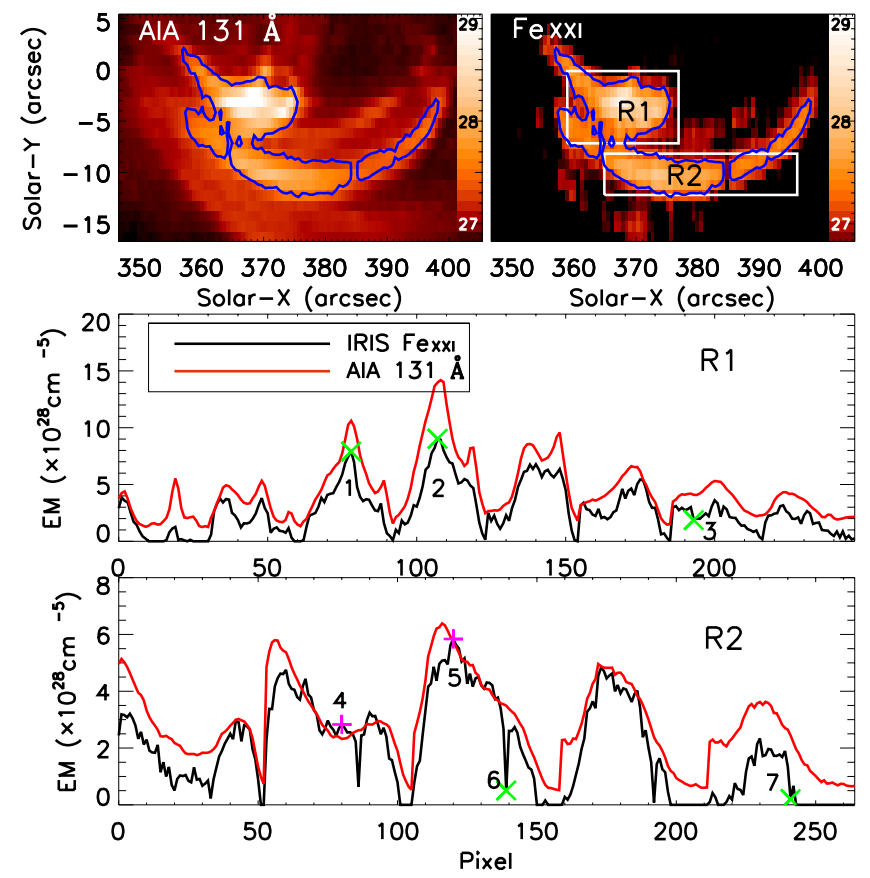

Fig. 16. Emission measures from AIA $131 \AA$ and IRIS Fe XXI at $11 \mathrm{MK}$ for the second flare. The two white boxes are the selected regions (R1 and R2). In the lower panels, the black line is the Fe XXI EM, and the red line is the 131 A EM. The +'s mark pixels where the Fe XXI EM is higher, and the $\times$ 's mark selected pixels, shown in Fig. 17 with higher 131 EM.

bright point on the edge of the kernels. The two points in the next highest intensity bin with a ratio below 0.5 are also from the edge of the kernel region. The other points with ratios less than 0.5 are from the bright western edge of the $131 \AA$ A region where both the Fe XXI and $131 \AA$ emission rapidly decreases. The higher ratio values are mostly from the lowest intensity bin on the edge of the loop regions. These may be due to cooler material on the edge of the loop or background $131 \AA$ emission. Therefore the typical loop value in flare 1 is the mean of the low-intensity bins, 0.55 or $81 \%$ Fe XXI.

The flare 2 loops have more scatter in their ratio. In the highest intensity bin, there are four points with a ratio around 0.6 (67\% Fe XXI). These are from the bright region at the center of R1. As mentioned, these points have blueshifted Fe XXI and at the same time are brightened in all AIA channels, suggesting that it is a site of chromospheric evaporation, not a typical loop. The scatter in the low-intensity bins is mostly caused by coalignment issues in the $\mathrm{R} 2$ region as can be seen in Fig. 16. The last row, around position 7 , produced many of the high ratio points. These points are along the edge of the loop and - like the edges of the loops in flare 1 - may be due to cooler material on the edge of the loop or background $131 \AA$ emission. In flare 2, the average value 0.55 (81\%) Fe XXI is typical for the $131 \AA$ loops.

The ratio in the kernels covers the full range from $0-100 \%$ Fe XXI. We have plotted the ratio as a function of both Fe XXI and 131 intensity. As the intensities increase the contribution of Fe XXI to the $131 \AA$ channel increases up to the value seen in the loops. In flare 1, as noted above and shown in Fig. 18, the Fe XXI EM is greater than the 131 EM in the brightest pixels. We attribute this to imperfect coalignment or different filling factors for the two instruments, so the ratio in the two highest intensity bins of flare 1 are not included in our final conclusions. Below a $131 \AA ̊$ intensity of $600 \mathrm{DN} \mathrm{s}^{-1}$, a significant fraction of pixels

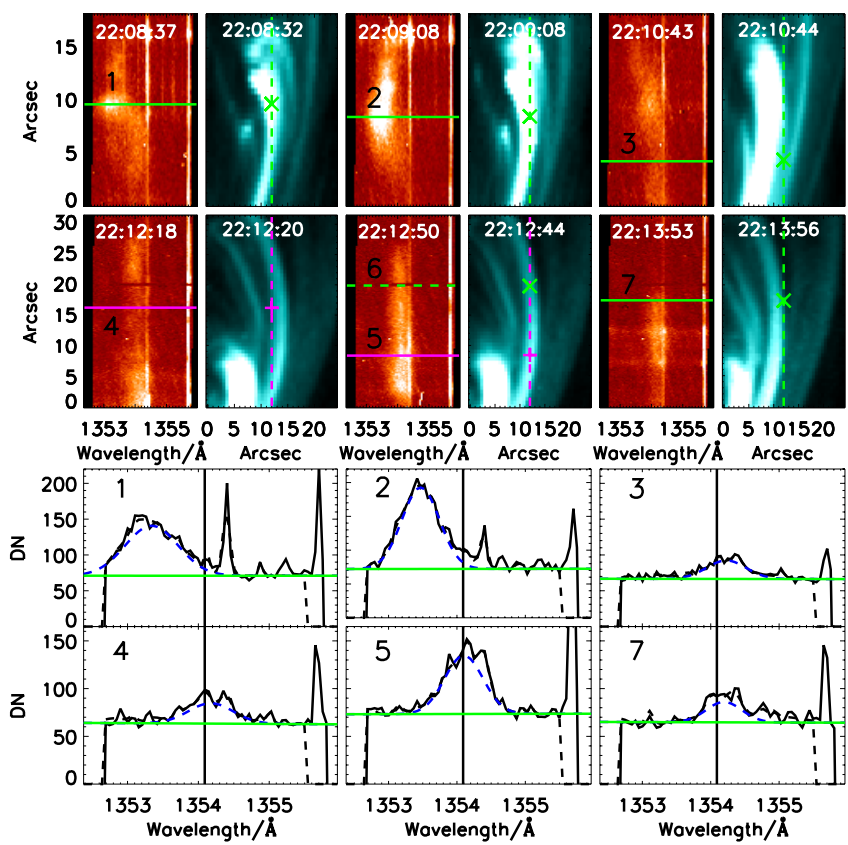

Fig. 17. Selected IRIS spectra and AIA intensities for the second flare. The vertical lines in the AIA images mark the positions of the IRIS slits. The numbers on the IRIS images show the positions of the selected points in Fig. 16. The +'s and X's in the AIA images mark the positions of these points in the AIA images. Underneath are the IRIS spectra (solid black line) and their fits (black dashed line). The green solid line is the background, the blue dashed line is background + Fe XXI line, while the vertical line is the rest wavelength of Fe XXI line.

have no Fe XXI emission. Thus, there is structure within the kernels on the scale of the AIA pixel size, $0.6^{\prime \prime}$. If the 131 pixels in the middle of the intensity range are considered, the ratio is between 0.55 and 0.75 (see Figs. 18 and 19), which implies that the 131 kernel EM is between 20-60\% greater than the Fe XXI EM. This broad range again implies a high-resolution structure in the kernels not seen in the $131 \AA$ images. If the continuum emission is about $20 \%$ of the Fe XXI as found by Milligan \& McElroy (2013), then our result implies that from zero to $52 \%$ of the flare kernel $131 \AA$ emission is due to cooler plasma emission.

These results assume that both instruments are well calibrated and the data are coaligned. The fact that we obtain a consistent value of $80 \% \mathrm{Fe}$ XXI contribution to the 131 channel in the loops suggests that the calibration is good. A $20 \%$ error in the intercalibration would lead to a $10 \%$ change in the Fe XXI contribution.

\section{Conclusions and discussion}

Using the high-resolution IRIS spectral data together with the SDO data, we study two solar flares in AR 11875 that occurred on 24 October 2013. We obtain the intensity of Fe XXI from IRIS data and compare it with AIA data at $131 \AA$. Coalignment to an accuracy of one AIA pixel (0.6") is achieved by coaligning the IRIS C I to AIA $1600 \AA$ (see Fig. 3) and the IRIS Fe XXI to AIA $131 \AA$ images. Fe XXI is a hot, broad line, which in flare kernels is blended with many cold lines. Previous studies (Doschek et al. 1975; Cheng et al. 1979; Mason et al. 1986) at lower resolution only consider blending of Fe XXI with the C I line. However, as IRIS reveals, there are several additional cold chromospheric lines blending with the Fe XXI (see Fig. 7). After studying other IRIS flare kernel spectra, we were able to identify 

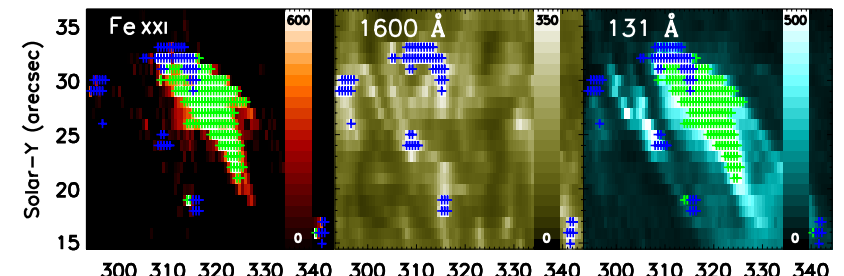

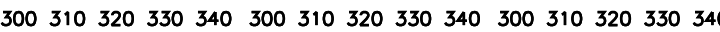
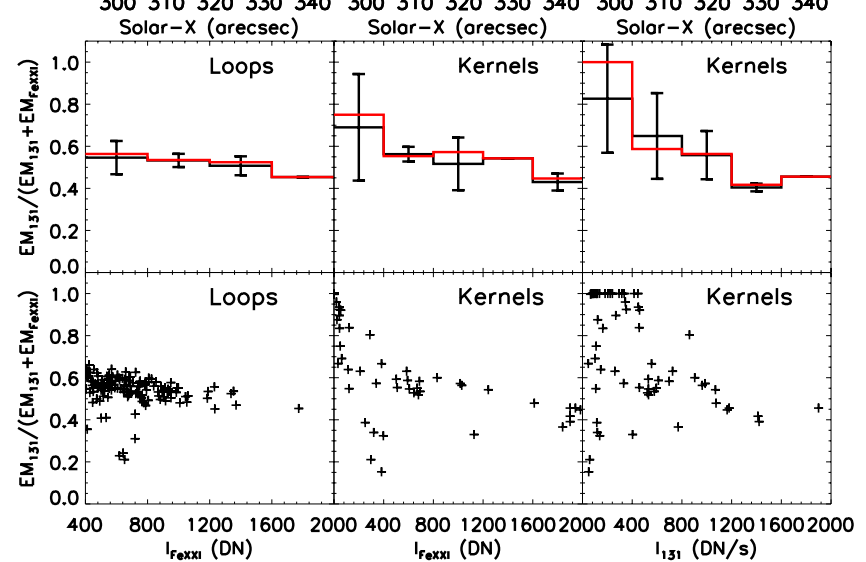

Fig. 18. Quantitative analysis of the Fe XXI and $131 \AA$ EMs for flare 1. The top row from left to right shows Fe XXI, $1600 \AA$, and $131 \AA$ intensity. The middle panels show the mean and median (red line) ratios $\mathrm{EM}(131) /(\mathrm{EM}(131)+\mathrm{EM}(\mathrm{Fe} \mathrm{XXI}))$ vs. Fe XXI intensity in loops (bin $=400 \mathrm{DN})$ and kernels $($ bin $=400 \mathrm{DN})$, and on the right the ratio $\operatorname{EM}(131) /(\operatorname{EM}(131)+\operatorname{EM}(F e X X I))$ vs. intensity of $131 \AA$ (bin = $400 \mathrm{DN} \mathrm{s}^{-1}$ ). The bottom panels show the same ratios vs. intensity but as scatter plots. In the loops the mean, standard deviation, and median ratios are $0.54 \pm 0.07$ and 0.55 .

lines in different parts of the IRIS spectrum that can be used to constrain the intensity, widths, and positions of the blending cold lines (see Table 1). Using 17 Gaussian lines and a linear continuum we are able to fit the spectra and obtain reliable Fe XXI intensities at all positions.

The spectra show regions at the onset of the flares where of Fe XXI is coincident with cold-line emission (see Figs. 10 and 12). We have carefully checked the fits to make sure that the procedure has worked at these positions (see Figs. 11 and 13). In these regions, the Fe XXI line shows high blueshifts up to $200 \mathrm{~km} \mathrm{~s}^{-1}$, which is the edge of the spectral window. This velocity is also consistent with other hot spectral lines seen in flares, such as Fe XIX (Czaykowska et al. 2001; Teriaca et al. 2003; Brosius \& Phillips 2004), Fe XXIII, and Fe XXIV (Milligan \& Dennis 2009). In our study, the EMs have a magnitude of about $10^{28} \mathrm{~cm}^{-5}$, although some pixels can reach $10^{29} \mathrm{~cm}^{-5}$ (see Figs. 14 and 16), which also agrees with others (Graham et al. 2013; Fletcher et al. 2013).

It is well known that the $131 \AA$ channel in AIA has contributions from the Fe XXI and the Fe VIII line. Brosius \& Holman (2012) list a number of other transition region lines that may also be present. We compare the EMs of AIA $131 \AA$ and the IRIS Fe XXI line pixel-by-pixel, assuming all emission is from hot plasma. For loop regions the AIA $131 \AA$ EMs are about $20 \%$ greater than the Fe XXI EMs (see Figs. 18 and 19). This is consistent with the analysis of Milligan \& McElroy (2013) that $20 \%$ of the $131 \AA$ channel emission is due to continuum in flares. We also identified a loop region with about $33 \%$ greater 131 than Fe XXI EM. Since, this region simultaneously brightened in all AIA channels and was associated with Fe XXI blueshifts, we attribute the lower-than-average Fe XXI to additional Fe VIII due to

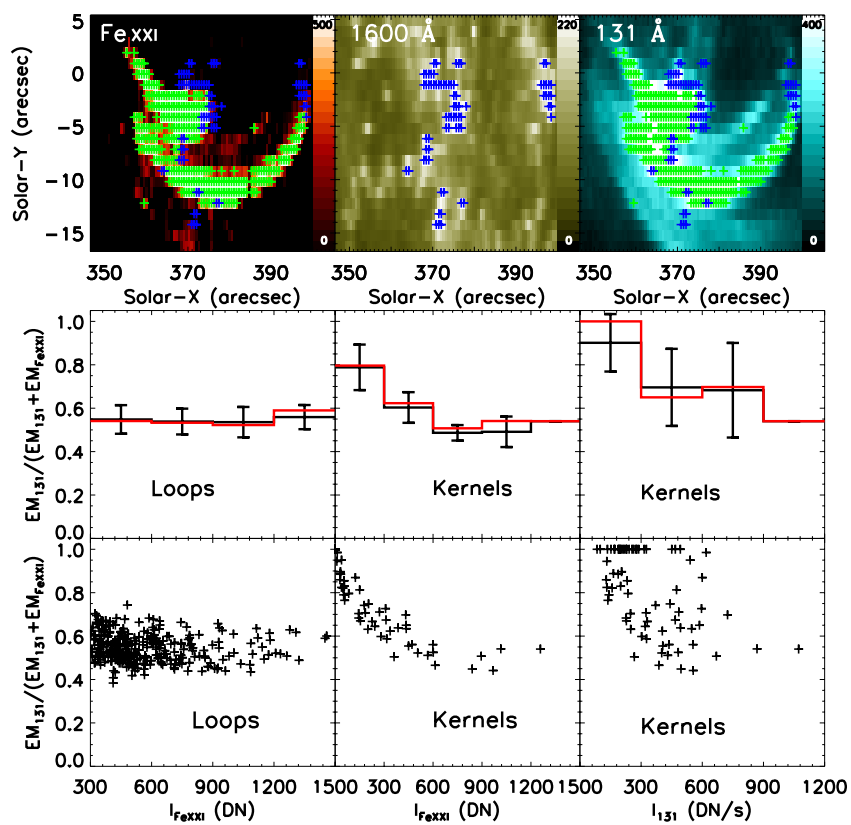

Fig. 19. Quantitative analysis of the Fe XXI and 131 EMs for flare 2. The top row from left to right shows FeXXI, $1600 \AA$, and $131 \AA$ intensity. The middle panels show the mean and median (red line) ratios $\mathrm{EM}(131) /(\mathrm{EM}(131)+\mathrm{EM}(\mathrm{Fe}$ XXI) $)$ vs. Fe XXI intensity in loops $($ bin $=300 \mathrm{DN})$ and kernels $($ bin $=300 \mathrm{DN})$, and on the right the ratio $\operatorname{EM}(131) /(\operatorname{EM}(131)+\operatorname{EM}(F e X X I))$ vs. intensity of $131 \AA$ (bin $=300 \mathrm{DN} \mathrm{s}^{-1}$ ). The bottom panels show the same ratios vs. intensity but as scatter plots. In the loops the mean, standard deviation, and median ratios are $0.55 \pm 0.06$ and 0.54 .

a recent injection of cool plasma into the loop. In the brighter 131 flare kernels ( $>600 \mathrm{DN} \mathrm{s}^{-1}$ ), the $131 \mathrm{EM}$ is about $20-60 \%$ more than the IRIS Fe XXI suggesting that there is a contribution of up to $52 \%$ from cool plasma emission (Brosius \& Holman 2012).

A small-scale kernel structure results in a broad range of 131/Fe XXI EM ratios and sharp gradients in IRIS Fe XXI emission at sites of molecular and transition region emission. At the start of the flare, consecutive raster steps showed a large increase in Fe XXI across the kernels indicating that the kernel structure is both temporal and spatial. Future work will focus on the analysis of flares obtained at higher spectral resolution with broader wavelength windows and higher temporal cadence to resolve the temporal/spatial ambiguities.

Acknowledgements. The authors would like to thank the anonymous referee for valuable comments that improved the manuscript. IRIS is a NASA small explorer mission developed and operated by LMSAL with mission operations executed at NASA Ames Research center and major contributions to down-link communications funded by the Norwegian Space Center (NSC, Norway) through an ESA PRODEX contract. We are indebted to the IRIS and SDO teams for providing the high-resolution data. We would also like to thank the people at MPS, in particular Professor Hardi Peter, Doctors Li, Leping, Chen, Feng and Guo, Lijia.

\section{References}

Antonucci, E., Gabriel, A. H., Acton, L. W., et al. 1982, Sol. Phys., 78, 107 Benz, A. O. 2008, Liv. Rev. Sol. Phys., 5, 1

Boerner, P., Edwards, C., Lemen, J., et al. 2012, Sol. Phys., 275, 41

Brosius, J. W., \& Holman, G. D. 2012, A\&A, 540, A24

Brosius, J. W., \& Phillips, K. J. H. 2004, ApJ, 613, 580

Cheng, C.-C., Feldman, U., \& Doschek, G. A. 1979, ApJ, 233, 736

Cheng, X., Ding, M. D., \& Fang, C. 2015, ApJ, 804, 82

Czaykowska, A., Alexander, D., \& De Pontieu, B. 2001, ApJ, 552, 849

De Pontieu, B., Title, A. M., Lemen, J. R., et al. 2014, Sol. Phys., 289, 2733 
DeRosa, M., \& Slater, G. 2013, Guide to SDO Data Analysis edited on February 19,2013

Doschek, G. A., Dere, K. P. Sandlin, G. D., et al. 1975, ApJ, 196, L83

Feldman, U., Curdt, W., Landi, E., \& Wilhelm, K. 2000, ApJ, 544, 508

Fletcher, L., \& Hudson, H. 2001, Sol. Phys., 204, 69

Fletcher, L., Pollock, J. A., \& Potts, H. E. 2004, Sol. Phys., 222, 279

Fletcher, L., Dennis, B. R., Hudson, H. S., et al. 2011, Space Sci. Rev., 159, 19

Fletcher, L., Hannah, I. G., Hudson, H. S., \& Innes, D. E. 2013, ApJ, 771, 104 Gan, W. Q., Li, Y. P., \& Miroshnichenko, L. I. 2008, Adv. Space Res., 41, 908 Graham, D. R., \& Cauzzi, G. 2015, ApJ, 807, L22

Graham, D. R., Hannah, I. G., Fletcher, L., \& Milligan, R. O. 2013, ApJ, 767, 83

Innes, D. E. 2001, A\&A, 378, 1067

Innes, D. E. 2008, A\&A, 481, L41

Kliem, B., Dammasch, I. E., Curdt, W., \& Wilhelm, K. 2002, ApJ, 568, L61

Landi, E., Young, P. R., Dere, K. P., Del Zanna, G., \& Mason, H. E. 2013, ApJ, 763,86

Lemen, J. R., Title, A. M., Akin, D. J., et al. 2012, Sol. Phys., 275, 17

Li, D., Ning, Z. J., \& Zhang, Q. M. 2015, ApJ, 807, 72
Mason, H. E., Shine, R. A., Gurman, J. B., \& Harrison, R. A. 1986, ApJ, 309, 435

McIntosh, S. W., De Pontieu, B., Hansteen, V., \& Boerner, P. 2013, A User's Guide To IRIS Data Retrieval, Reduction and Analysis, October, 30 Milligan, R. O., \& Dennis, B. R. 2009, ApJ, 699, 968

Milligan, R. O., \& McElroy, S. A. 2013, ApJ, 777, 12

Milligan, R. O., Gallagher, P. T., Mathioudakis, M., et al. 2006, ApJ, 638, L117 Ning, Z., \& Cao, W. 2011, Sol. Phys., 269, 283

O’Dwyer, B., Del Zanna, G., Mason, H. E., Weber, M. A., \& Tripathi, D. 2010, A\&A, 521, A21

Qiu, J., Lee, J., Gary, D. E., \& Wang, H. 2002, ApJ, 565, 1335

Teriaca, L., Falchi, A., Cauzzi, G., et al. 2003, ApJ, 588, 596

Teriaca, L., Falchi, A., Falciani, R., Cauzzi, G., \& Maltagliati, L. 2006, A\&A, 455,1123

Testa, P., Drake, J. J., \& Landi, E. 2012, ApJ, 745, 111

Tian, H., DeLuca, E., \& Reeves, K. K., et al. 2014, ApJ, 786, 137

Tian, H., Young, P. R., Reeves, K. K., et al. 2015, ApJ, 811, 139

Wang, T. J., Solanki, S. K., Curdt, W., et al. 2003, A\&A, 406, 1105

Young, P. R., Doschek, G. A., Warren, H. P., \& Hara, H. 2013, ApJ, 766, 127

Young, P. R., Tian, H., \& Jaeggli, S. 2015, ApJ, 799, 218 